\title{
Sampling theory for linear integral transforms
}

\author{
Robert J. Marks II \\ Department of Electrical Engineering F'T-10, University of Washington, Seattle, Washington 98195
}

Received July 28, 1980; revised manuscript received October 17, 1980

\begin{abstract}
A sampling theorem is developed to reduce integration error in matrix-vector and linear multiplexing processors that perform discrete versions of continuous linear operations. By simply filtering the operation kernel before sampling, one can perform integration-error-free processing on inputs sampled at their Nyquist rate. Example applications to Laplace and Hilbert transformation are presented.
\end{abstract}

\section{Introduction}

Much recent attention has been focused on optical implementation of the general linear operation

$$
g(x)=\int_{-\infty}^{\infty} u(\xi) h(x ; \xi) \mathrm{d} \xi
$$

in both one and two dimensions. The idea is to design a processor that, when fed an input $u(\cdot)$, will yield the corresponding output $g(\cdot)$. Specific cases of Eq. (1) are numerous and include correlation, convolution, and linear integral transformations (Laplace, Abel, Mellin, Hilbert, Hankel, etc.).

One popular approach is to evaluate Eq. (1) by trapezoidal integration:

$$
g(x) \approx \Delta \sum_{n} u(n \Delta) h(x ; n \Delta),
$$

where $\Delta$ is the input sampling interval. If the output is sampled, Eq. (2) can be expressed simply as a matrix-vector product.

Coherent and noncoherent optical implementations of the matrix-vector operation in Eq. (2) are numerous. ${ }^{1,2}$ Bocker ${ }^{3}$ designed a system utilizing scanning mirrors and vidicons. Shadow-casting processors have been developed by Schneider and Fink ${ }^{4}$ as well as by Tamura and Wyant. ${ }^{5}$ Monahan et al. ${ }^{6}$ use chargecoupled devices as storage and shift elements in their optical processor. Goodman and Dias 7,8 have recently developed another incoherent processor with incredible information-throughput capacity.

Optical implementations akin to Eq. (2) have also been carried out in analog form. That is, the output is not sampled. Krile et al. ${ }^{9,10}$ and Jones et al. ${ }^{11}$ multiplexed a number of terms on a single hologram using phase-coded reference beams. Various processors using temporal holographic integration have been presented by Marks. ${ }^{12}$ Kasturi et al. ${ }^{13}$ use a frequency-domain multiplexing scheme.

For certain systems whose kernels (impulse response) $h$ conform to certain band-limiting criteria, Eq. (2) can be shown to be exact. ${ }^{14-16}$ There are, however, two major drawbacks. First of all, the input and the kernel must be sampled in excess of their respective Nyquist rates. Second, and more importantly, a vast number of commonly used linear operations do not conform to the band-limited criteria.

The purpose of this Letter is to show that by a simple alteration of the impulse response in Eq. (1), the expression in Eq. (2) can be made exact in the spirit of the sampling theorem. The revised expression can be utilized in the above processors to reduce error that is due to trapezoidal-integration approximation. Also, certain linear operations that cannot be directly evaluated by use of Eq. (2) because of singularities are shown to be capable of implementation through the sampling theorem characterization. As will be demonstrated, the sole requirements are

1. The band-limited input has finite energy.

2. The input samples are unaliased.

3. The kernel is well behaved.

\section{Development}

For clarity of presentation, we will assume that the unaliased input data come from a band-limited signal of the low-pass type. Such signals are unaltered by low-pass filtering. Thus

$$
u(\xi)=2 B \int_{-\infty}^{\infty} u(\eta) \operatorname{sinc} 2 B(\xi-\eta) \mathrm{d} \eta,
$$

where $\operatorname{sinc} x=\sin \pi x /(\pi \mathrm{x})$,

$$
B \geq W,
$$

and $W$ is the maximum frequency component of $u$. Substituting into Eq. (1) gives

$$
g(x)=\int_{-\infty}^{\infty} u(\eta) \hat{h}(x ; \eta) \mathrm{d} \eta,
$$

where the low-passed kernel (LPK) is

$$
\hat{h}(x ; \eta)=2 B \int_{-\infty}^{\infty} h(x ; \xi) \operatorname{sinc} 2 B(\xi-\eta) \mathrm{d} \xi .
$$

Even though the kernel in Eq. (4) is altered, it yields the same result as in Eq. (1).

Since both the input and the LPK are band limited in $\eta$, they can be expressed by the uniformly converging Whittaker-Shannon sampling theorem ${ }^{17}$ : 


$$
\begin{gathered}
u(\eta)=\sum_{n=-\infty}^{\infty} u(n \Delta) \operatorname{sinc}(n-\eta / \Delta), \\
h(x ; \eta)=\sum_{m=-\infty}^{\infty} \hat{h}(x ; m \Delta) \operatorname{sinc}(m-\eta / \Delta),
\end{gathered}
$$

where the input sampling interval must be chosen such that

$$
\Delta \leq 1 / 2 B \leq 1 / 2 W .
$$

Substituting Eqs. (6a) and (6b) into Eq. (4) gives

$$
\begin{aligned}
g(x)= & \sum_{n} \sum_{m} u(n \Delta) \hat{h}(x ; m \Delta) \\
& \times \int_{-\infty}^{\infty} \operatorname{sinc}(n-\eta / \Delta) \operatorname{sinc}(m-\eta / \Delta) \mathrm{d} \eta \\
= & \Delta \sum_{n} u(n \Delta) \hat{h}(x ; n \Delta) .
\end{aligned}
$$

This is the desired result. Comparing it with Eq. (2), we conclude that the inaccuracy that is due to trapezoidal integration can be totally eliminated if the LPK is used in lieu of the original kernel.

A sufficient condition for Eq. (8) to converge for a given value of $x$ is that both $u$ and $h$ be square integrable in $\eta$. That is

$$
E_{u}=\int_{-\infty}^{\infty}|u(\eta)|^{2} \mathrm{~d} \eta=\Delta \sum_{n=-\infty}^{\infty}|u(n \Delta)|^{2}<\infty
$$

and

$$
E_{h}(x)=\int_{-\infty}^{\infty}|\hat{h}(x ; \eta)|^{2} \mathrm{~d} \eta=\Delta \sum_{n=-\infty}^{\infty}|\hat{h}(x ; n \Delta)|^{2}<\infty .
$$

Then, from Schwarz's inequality,

$$
|g(x)|^{2} \leq E_{u} E_{h}(x)
$$

\section{Examples}

To illustrate use of Eq. (8), we now present example applications for the cases of Laplace and Hilbert transformation.

\section{Laplace Transform}

The Laplace transform can be written as

$$
g(x)=\int_{0}^{\infty} u(\xi) e^{-x \xi} \mathrm{d} \xi
$$

Comparing with Eqs. (1) and (4), we have

$$
h(x ; \xi)=e^{-x \xi} \mu(\xi)
$$

and

$$
\hat{h}(x ; \eta)=\int_{0}^{\infty} e^{-x \xi} \operatorname{sinc}(\xi-\eta) \mathrm{d} \xi,
$$

where $\mu(\cdot)$ denotes the unit step and we have chosen $2 B$ $=1$.

Table 1. Laplace Transform of $u(\xi)=(d / d \xi) \sin \pi \xi / \xi$

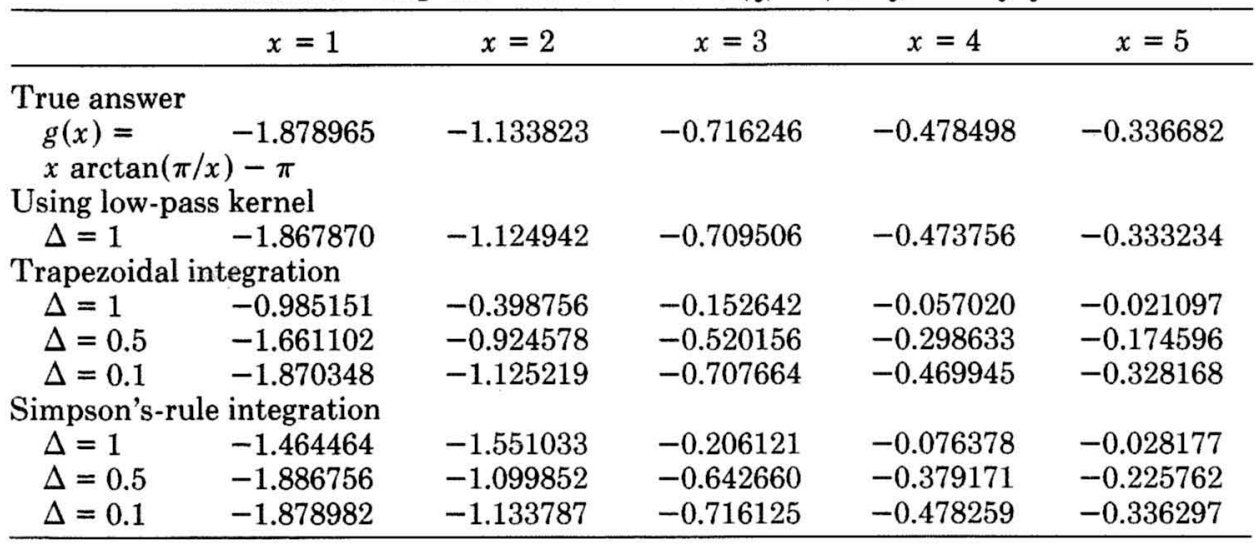

Table 2. Laplace Transform of $u(\xi)=[\sin (\pi \xi / 2) / \xi]^{2}$

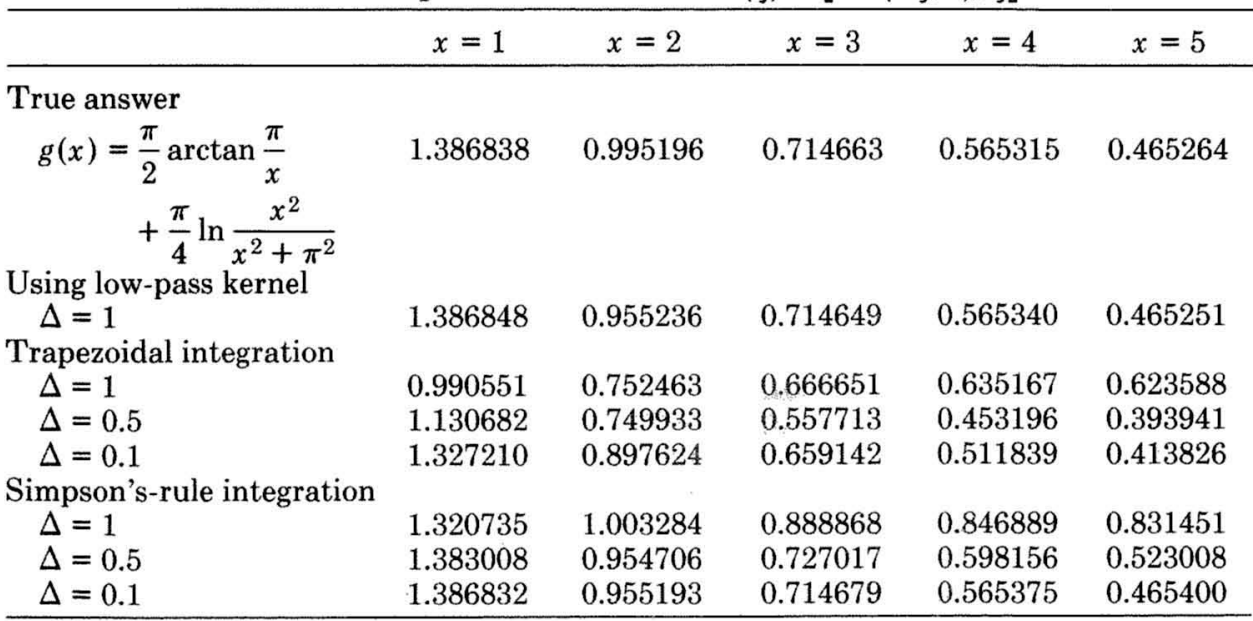


Numerical results for two band-limited functions are given in Tables 1 and 2 . For both examples, $2 W=1$. From the results shown, we observe that trapezoidal integration does not give so accurate a result even at 10 times the sampling rate. The more accurate Simpson's-rule integration results are also seen to be inferior to the LPK technique. The error in the LPK results stems primarily from error in the digital integration of Eq. (9), which was performed on an HP-41C programmable calculator.

\section{Hilbert Transformation}

The Hilbert transform

$$
g(x)=-\frac{1}{\pi} \int_{-\infty}^{\infty} \frac{u(\xi) \mathrm{d} \xi}{x-\xi}
$$

cannot be accurately evaluated by direct trapezoidal integration because of the singularity at $\xi=x$. Fourier-transform analysis, rather, is commonly used. We will now show, however, that through application of the LPK, an accurate matrix-vector characterization of the Hilbert transform is possible. From Eq. (10),

$$
h(x ; \xi)=\frac{-1}{\pi(x-\xi)} .
$$

If we choose $B=W$, the corresponding LPK is

$$
\begin{aligned}
\hat{h}(x ; \eta) & =\int_{-W}^{W}\left[-\frac{1}{\pi} \int_{-\infty}^{\infty} \frac{\exp (-j 2 \pi f \xi)}{x-\xi} \mathrm{d} \xi\right] e^{j 2 \pi f \eta} \mathrm{d} f \\
& =-j \int_{-W}^{W} \operatorname{sgn} f e^{-j 2 \pi f(x-\eta)} \mathrm{d} f \\
& =-2 W \sin \pi W(x-\eta) \operatorname{sinc} W(x-\eta)
\end{aligned}
$$

A further simplification arises after we note that Hilbert transformation is a shift-invariant operation. Thus, if $u$ is band limited, so then is the output, $g$. This being true, we need to know $g$ only at the points where $x=m / 2 W$. Substituting Eq. (11) into Eq. (8) with $\Delta$ $=1 / 2 W$ gives

$$
g(m \Delta)=\sum_{n} u(n \Delta) \sin \frac{\pi}{2}(m-n) \operatorname{sinc} \frac{1}{2}(m-n) .
$$

Noting that every other term is zero yields the final desired result,

$$
g(m \Delta)=\frac{-2}{\pi} \sum_{m-n \text { odd }} \frac{u(n \Delta)}{m-n} .
$$

This matrix-vector version of the Hilbert transform contains no singularities and is exact for all band-limited inputs.

\section{Remarks}

The sampling theorem in Eq. (8) is superior to that applicable to variation-limited operations ${ }^{14-16}$ simply because required sampling rates are lower and the LPK method is applicable to a larger operation class-including variation-limited operations.

Last, we note--as the reader can easily verify - that the LPK method does not yield any computational insight into discrete Fourier transformation.

\section{References}

1. M. A. Monahan, K. Bromley, and R. P. Bocker, "Incoherent optical correlators," Proc. IEEE 65, 121 (1977).

2. J. F. Walkup, "Space-variant coherent optical processing," Opt. Eng. 19, 339 (1980).

3. R. P. Bocker, "Matrix multipication using incoherent optical techniques," Appl. Opt. 13, 1670 (1974).

4. W. Schneider and W. Fink, "Incoherent optical matrix multiplication," Opt. Acta 22, 879 (1975).

5. P. N. Tamura and J. C. Wyant, "Two-dimensional matrix multiplication using coherent optical techniques," Opt. Eng. 18, 198 (1979).

6. M. A. Monahan et al., "Incoherent electro-optical processing with CCD's," in Proceedings of Digital International Computing Conference (Institute of Electrical and Electronics Engineers, New York, 1975).

7. A. R. Dias, "Incoherent matrix-vector multiplication for high-speed data processing," Ph.D. thesis (Stanford U. Press, Stanford, Calif., 1980).

8. J. W. Goodman, "The matrix-vector multiplication incoherent processor," presented at Workshop on Future Directions for Optical Information Processing, May 20-22, 1980, Lubbock, Texas.

9. T. F. Krile et al., "Holographic representation of spacevariant systems using phase-coded reference beams," Appl. Opt. 16, 3131 (1977).

10. T. F. Krile et al., "Multiplex holography with chirpmodulated binary phase-coded reference-beam masks," Appl. Opt. 18, 52 (1979).

11. M. I. Jones, J. F. Walkup, and M. O. Hagler, "Multiplex holography for space-variant optical computing," Proc. Soc. Photo-Opt. Instrum. Eng. 177, 16 (1979).

12. R. J. Marks II, "Two-dimensional space-variant processing using temporal holography: processor theory," Appl. Opt. 18, 3670 (1979).

13. R. Kasturi, T. F. Krile, and J. F. Walkup, "Space-variant 2-D processing using a sampled input/sampled transfer function approach," presented at 1980 International Optical Computing Conference, April 7-11, 1980, Washington, D.C.

14. R. J. Marks II, J. F. Walkup, and M. O. Hagler, "A sampling theorem for space-variant systems," J. Opt. Soc. Am. 66, 918 (1976).

15. R. J. Marks II, J. F. Walkup, and M. O. Hagler, "Sampling theorems for linear shift-variant systems," IEEE Trans. Circuits Syst. CAS-25, 228 (1978).

16. R. J. Marks II, J. F. Walkup, and M. O. Hagler, "Methods of linear system characterization through response cataloging," Appl. Opt. 18, 655 (1979).

17. J. W. Goodman, Introduction to Fourier Optics (McGraw-Hill, New York, 1968). 